\title{
A marked decrease in heart rate variability in Marfan syndrome patients with confirmed FBN1 mutations
}

\author{
Andriy Cherkas ${ }^{1}$, Rustam Zhuraev $^{2}$ \\ ${ }^{1}$ Department of Medicine, Lviv College of Physical Culture, Lviv, Ukraine \\ ${ }^{2}$ Institute of Clinical Pathology, Lviv National Medical University, Lviv, Ukraine
}

\begin{abstract}
Background: The studies on heart rate variability (HRV), a key predictor of all-cause mortality, in Marfan syndrome (MS), up to now have not been reported, especially in patients with FBN1 mutations.
\end{abstract}

Methods: Among 18 MS patients with the phenotype of MS meeting inclusion criteria 15 have had a FBN1 gene mutation. Short electrocardiography records were taken in the supine position and during orthostatic tests. The control group consisted of 30 apparently healthy nonathletes matched by age and gender.

Results: Heart rates in MS patients with the FBN1 mutation were increased in both the supine position and orthostatic test $(p<0.001)$. Most of the time-domain (standard deviation, pNN50) and frequency-domain (total power, very low, low, and high frequency) parameters of $H R V$ were significantly reduced in the MS patients $(p<0.001)$.

Conclusions: A marked decrease in HRV, documented in the study, may be an important clinical feature in MS patients with confirmed FBN1 gene mutations. (Cardiol J 2016; 23, 1: 23-33)

Key words: Marfan syndrome, FBN1 mutation, heart rate variability, heart rate, autonomic control, transforming growth factor beta, metabolism

\section{Introduction}

Marfan syndrome (MS), being a relatively common genetic disease with an estimated population incidence $2-3 / 10,000$, raises serious concerns due to aortal dilation that significantly increases risk of dissection, with potentially life threatening complications often developing at a young age $[1,2]$. Other major clinical manifestations include typical abnormalities of the skeleton, eyes and a number of other health problems $[2,3]$.
The molecular basis of this disease is the mutation of the FBN1 gene, encoding for the synthesis of fibrillin 1 , a protein providing important structural function in the extracellular matrix. In addition, it participates in signaling pathways by transforming factor beta (TGF- $\beta$ ) binding domains. Therefore, altered function of FBN1 in MS leads to both structural (mucoid degeneration and degradation of the extracellular matrix) and functional (activation of the TGF- $\beta$ pathway) consequences [2]. Defective FBN1 structure is associated with activa-

Address for correspondence: Andriy Cherkas, MD, PhD, Chief of the Department of Medicine, Lviv College of Physical Culture, 1 Knyagyni Olgy St., Lviv, 79044 Ukraine, tel: +380322382794 (office), +380673415426 (cell), fax: +380322382793, e-mail: cherkasandriy@yahoo.com 
tion of proteases in response to gradual functional tissue damage together with TGF- $\beta$-related reparative responses leading occasionally to induction of apoptosis in cells. Also, focal sclerosis create the medium for development of clinical manifestations [4]. Besides, the strategy to block TGF- $\beta$ signaling ameliorated myocardial fibrosis and diastolic dysfunction in a model study [5], confirming the deteriorating effects of excessive TGF- $\beta$.

Despite the wide presence of the FBN1 protein in various tissues, the noticeable changes develop primarily in loci where the function of this protein is critical - aorta, eyes, skeleton, visceral layer of pleura etc. [1-3]. Naturally, the attention of investigators is focused mainly on the major pathological changes, while many other aspects of the disease remain unstudied. In particular the heart rhythm peculiarities and abnormalities in MS are less studied. In fact, these changes seem to be clinically not as important as aortal dissection or lens ectopy. However, proper understanding of heart rhythm changes eventually appearing in MS patients may improve the diagnostics and potential risk assessment. Finally, it could be valuable in the search of long-term follow-up and the criteria for the evaluation of management efficiency in this group of patients.

The most significant heart rhythm abnormalities previously reported in MS are interval PQ prolongation (decreased atrioventricular conductivity) and ventricular extrasystoles [6]. Since 1996, when standards of heart rate variability (HRV) measurements and interpretation were published [7], dramatic growth of interest in the method has been observed. The consensus exists that the parameters of HRV reflect the activities of sympathetic and parasympathetic branches of the autonomic nervous system. Among the most important associations of reduced HRV reported to the date are all cause mortality, risk of fatal arrhythmias, and many others [8]. In addition, the relationships of HRV to important inflammatory parameters as well as insulin resistance, oxidative stress and dyslipidemia have been established [9, 10].

To our best knowledge, to date there have been no reports of HRV parameters in MS patients derived from the short electrocardiography (ECG) records taken in laboratory conditions. The only known study of Holter heart monitoring of MS patients which includes HRV parameters has recently been published [11]. However, in the paper, there is a lack of HRV description. The other study from this group includes HRV data from $24 \mathrm{~h}$ monitoring. However, this paper focused on stratification of sudden cardiac death in MS, and the HRV was compared between two subgroups of patients without indication if it was high or low compared to healthy subjects [12]. There are also two papers reporting increased fatigue and orthostatic intolerance in MS patients. Authors suggest that it is possibly associated with increased body height and structural abnormalities of blood vessels, while autonomic function or HRV was not assessed [13, 14]. Therefore, the aim of this work is to study the HRV parameters in a cohort of MS patients with confirmed FBN1 mutations and to compare them to an age and gender matched control group. In addition, an effort has been made to analyze the role of possible important factors contributing to the changes in HRV parameters in MS patients.

\section{Methods}

\section{Patients and clinical data}

A total number of 19 MS patients were recruited for this study (case-control design) from 2010 to 2014 . The median age was $30.7 \pm 1.6$ (range 23-42) years. All patients met strict diagnostic criteria for MS based on the original revised Ghent nosology [3] and respective Systemic score was determined by means of an online tool at http:// $/ /$ www.marfan.org/dx/score. All the patients underwent physical examination, routine clinical tests and echocardiography. Height was measured with a standardized stadiometer, patients were weighed on electronic scales (OMRON Corporation, Kyoto, Japan), and body mass index (BMI) was calculated. Clinical Systemic scores were above 7 in majority of MS patients 11 of the MS patients (57.9\%). Nine $(47.4 \%)$ patients had aortic root/valve-replacing surgery (Bentall procedure) in the past. Patients with sinus rhythm were included in the study, while any heart rhythm disorders possibly significantly distorting HRV evaluation (e.g. atrial fibrillation; multiple extrasystoles, more than $1 / \mathrm{min}$, atrioventricular blocks of second and third degree etc.) were excluded from the study. We did not find any clinically significant heart conduction abnormalities like PR interval prolongation over $0.2 \mathrm{~s}$, or QT interval prolongation in MS patients and healthy subjects involved in the study. While recruiting $1 \mathrm{MS}$ patient was observed with frequent ventricular extrasystoles, therefore, his results were excluded from the database. Standard treatment of MS patients included losartan $100.0 \mathrm{mg}$ twice daily, however most of the patients were not taking this drugs or took it occasionally before participation in the study. Patients after Bentall surgery were 
taking warfarin in addition to standard treatment. The control group consisted of 30 apparently healthy non-athletes matched by age (range 20-40, mean $29.3 \pm 1.0$ years) and gender distribution (males/females ratio 12/7 in MS group vs. 20/10 in control group). The Ethics Committee of Lviv National Medical University approved the clinical and genetic program for MS. A written informed consent form was obtained from all patients. Data on familial and de novo cases was derived from family screening and tracing of clinical records.

\section{Genetic studies}

A Haploplex (Agilent Technologies, Santa Clara, California, USA) assay was used for enrichment of 12 TAA genes and the samples sequenced with next generation sequencing on a Miseq (Illumina, San Diego, California USA). Subsequently, the putative mutations were confirmed with Sanger sequencing on an ABI sequencer (Life Technologies, Carlsbad, California, USA). Patients were screened for FBN1, TGFBR1, TGFBR2, NOTCH1, FLNA, EFEMP2, SLC2A10, MYLK, MYH11, COL3A1, SMAD3 and ACTA2 genes. Genetic testing was performed in the Center of Medical Genetics, Antwerp, Belgium.

In the studied cohort of MS patients, we did not identify any other mutations than FBN1, which provided relative homogeneity of the group in terms of presence of identical biochemical changes caused by specific molecular defect. The other 3 patients had neither FBN1 nor any other mutations mentioned above, according to the screening results.

\section{Study of HRV}

During quiet wakefulness in the morning hours, not less than $24 \mathrm{~h}$ after the last significant physical exercise, the short-time records of ECG were taken. A computer electrocardiograph VNS-Micro (Neurosoft ${ }^{\circledast}$, Ivanovo, Russia) was used for ECG records. After 20 min of rest, studied subjects were asked to stay supine quietly for $5 \mathrm{~min}$ to ensure quasi-stationary condition HRV recording; afterwards they were asked to stand up rapidly and remain in the standing position for 6 min - active orthostatic test (OT). RR intervals were determined with a sampling frequency of $2.0 \mathrm{kHz}$ and were analyzed with Poly-Spectrum (Neurosoft ${ }^{\oplus}$, Ivanovo, Russia) software designed in line with HRV standards [7]. The time-domain parameters - standard deviation of normal RR intervals (SDNN), the square root of the mean squared differences of successive RR intervals (RMSSD), and percentage of differences between adjacent normal RR intervals exceeding 50 ms (pNN50) were determined. The power spectral analysis was performed sequentially with a fast Fourier transformation. The following frequency-domain variables were studied: total power (TP, $0.01-0.40 \mathrm{~Hz}$ ), high frequency power $(\mathrm{HF}, 0.15-0.40 \mathrm{~Hz})$, low frequency power (LF, $0.04-0.15 \mathrm{~Hz}$ ), and very low frequency power (VLF, 0.01-0.04 Hz). Occasional extrasystoles (not more than $1 / \mathrm{min}$ ) as well as artifacts were detected $a d$ oculus by an experienced physician and removed manually from the analysis. Extrasystoles were excluded from the analysis together with consecutive compensatory pause.

Short-term 5 min records of ECG for HRV analysis, despite some limitations, can be performed in strict laboratory conditions and provide nearly stationary (quasi-stationary) heart rate (HR), which is sufficient for accurate determination of HRV parameters according to the standards, in particular HF and LF spectral power. Short records, in fact, are suboptimal for SDNN and VLF determination, however in a 24 -h monitoring, a significant share of the values of these parameters account for day/night HR differences. Besides, careful OT evaluation can be performed only in laboratory conditions, which is important advantage over the 24-h Holter ECG monitoring, which is also often used for determination of HRV [15].

\section{Statistical analysis}

All data were processed using the statistical package Statistica 10.0 (Statsoft, Tulsa, Oklahoma, USA). Normal distribution of the obtained data was confirmed with Shapiro-Wilk's W-test. Normally distributed data is presented as means \pm standard error mean and abnormally distributed parameters (most of the parameters of HRV) as median (quartile range). Nonparametric Mann-Whitney U-test was used to compare HRV parameters, $\chi^{2}$ test was used for qualitative data presented as positive/ /negative. In figures, data is presented as mean and standard error mean (error bar). If not indicated otherwise, $\mathrm{p}<0.05$ was considered significant.

\section{Results}

\section{Anthropometric data}

There were $12(63.2 \%)$ male and $7(36.8 \%)$ female patients in this study. A control group consisted of 30 apparently healthy subjects of about the same age (Table 1) and similar gender distribution $-20(66.7 \%)$ males and 10 (33.3\%) females, difference with MS group not significant with $\mathrm{p}=0.802$ 
Table 1. Age and main anthropometric parameters of studied subjects (mean \pm standard error).

\begin{tabular}{lccc}
\hline Parameter & $\begin{array}{c}\text { Marfan syndrome } \\
\text { group }(\mathbf{n}=\mathbf{1 8})\end{array}$ & $\begin{array}{c}\text { Control group } \\
(\mathbf{n}=\mathbf{3 0})\end{array}$ & P value, t-test \\
\hline Age [years] & $30.7 \pm 1.6$ & $29.3 \pm 1.0$ & 0.431 \\
Height $[\mathrm{cm}]$ & $187.3 \pm 2.5$ & $178.4 \pm 1.1$ & $<0.001$ \\
Weight $[\mathrm{kg}]$ & $72.8 \pm 3.6$ & $78.8 \pm 2.4$ & 0.168 \\
Body mass index $\left[\mathrm{kg} / \mathrm{m}^{2}\right]$ & $20.6 \pm 0.8$ & $24.7 \pm 0.7$ & 0.001 \\
\hline
\end{tabular}

( $\chi^{2}$ test). Among the 19 MS subjects enrolled in the study, 15 have had confirmed FBN1 mutations by genetic studies. The data from these 15 patients were used for further statistical evaluations. One of the patients was excluded from the study since frequent ventricular extrasystoles were detected. Three remaining patients with clinical evidence of MS but without confirmed FBN1 mutation are discussed separately below in the text.

The comparison of anthropometric parameters of MS and control group is presented in Table 1. As it could be expected MS patients are taller and relatively slimmer (BMI is lower). These features are among the typical characteristics of MS.

\section{HRV in supine position}

The parameters of HRV in MS patients were found to have a specific pattern - increased HR with most of the HRV parameters decreased. HR in MS patients was significantly higher than in the controls 71.7 vs. 61.9 bpm (p < 0.001, Fig. 1A). All the time domain parameters including SDNN, RMSSD and pNN50 were found to be significantly lower in MS patients (Fig. 1B-D). SDNN and RMSSD is decreased roughly $2-3$-fold while pNN50 (reflecting mostly parasympathetic activity) decrease was much more profound - almost 15 -fold (Mann-Whitney U-test $\mathrm{p}<0.001$ in all cases).

The evaluation of the frequency domain HRV is also characterized by a dramatic decrease of these parameters in MS patients with FBN1 mutations (Fig. 2). TP is 4-fold lower than in the control group $(\mathrm{p}<0.001)$. Interestingly, the decrease of VLF, which is usually associated with the activity of humoral, metabolic and central regulatory influences on the heart rhythm, is not so dramatic - 2.8-fold ( $<<0.001)$, while LF (associated with mostly sympathetic output) decreased 5 -fold $(\mathrm{p}<0.001)$ and $\mathrm{HF}$ (mainly parasympathetic activity) decrease was even more profound -8 -fold $(\mathrm{p}<0.001)$. Despite such convincing evidence of changes in all frequency domain indexes of HRV the mean of $\mathrm{HF} / \mathrm{LF}$ ratio was not significantly dif- ferent in studied groups -1.92 vs. 1.84 in MS and control group, respectively ( $\mathrm{p}=0.279$, Mann-Whitney U-test), mainly due to high variations of this parameter within the groups.

Even more interesting seems to be the pattern of VLF-LF-HF distribution within the TP in MS patients with FBN1 mutation in comparison with the control group. While in the control group mean values of VLF, LF and HF power are almost equal with slight dominance of power of "autonomic" regulation (each LF and HF accounts for approximately $34 \%$ of TP), in MS patients, in addition to the decrease described above these frequency bands striking decrease in their share in TP (29 and $19 \%$ in LF and HF, respectively) was observed. Meanwhile the share of VLF in TP increased to $52 \%$ indicating "centralization" (relative increase of central nervous system involvement) of heart rhythm regulation in MS patients with FBN1 mutations (Fig. 3).

\section{HRV in orthostatic test}

In the OT, general characteristics of HRV remain similar to those in the supine position; however some important peculiarities have been revealed. HR increase in response to an active OT was similar in both the MS and the control group, HR was higher in patients $(\mathrm{p}=0.020$, Fig. $4 \mathrm{~A})$. Both SDNN and RMSSD in healthy controls were higher than in MS patients, $p<0.001$ (Fig. 4B, C). However, in the case of pNN50, no significant difference was found by t-test ( $\mathrm{p}=0.312$, Fig. $4 \mathrm{D})$. The later probably is due to relatively high values of standard deviation and standard error mean, which is a rather technical issue (method limitation) in this case. Interestingly, when pNN50 difference was compared by Mann-Whitney U-test, the difference was found to be significant $(\mathrm{p}<0.001)$.

HF waves are naturally suppressed in the orthostatic position and activation of LF band which is associated with sympathetic activity was observed. Absolute values and distribution patterns of other frequency domain HRV parameters are very simi- 

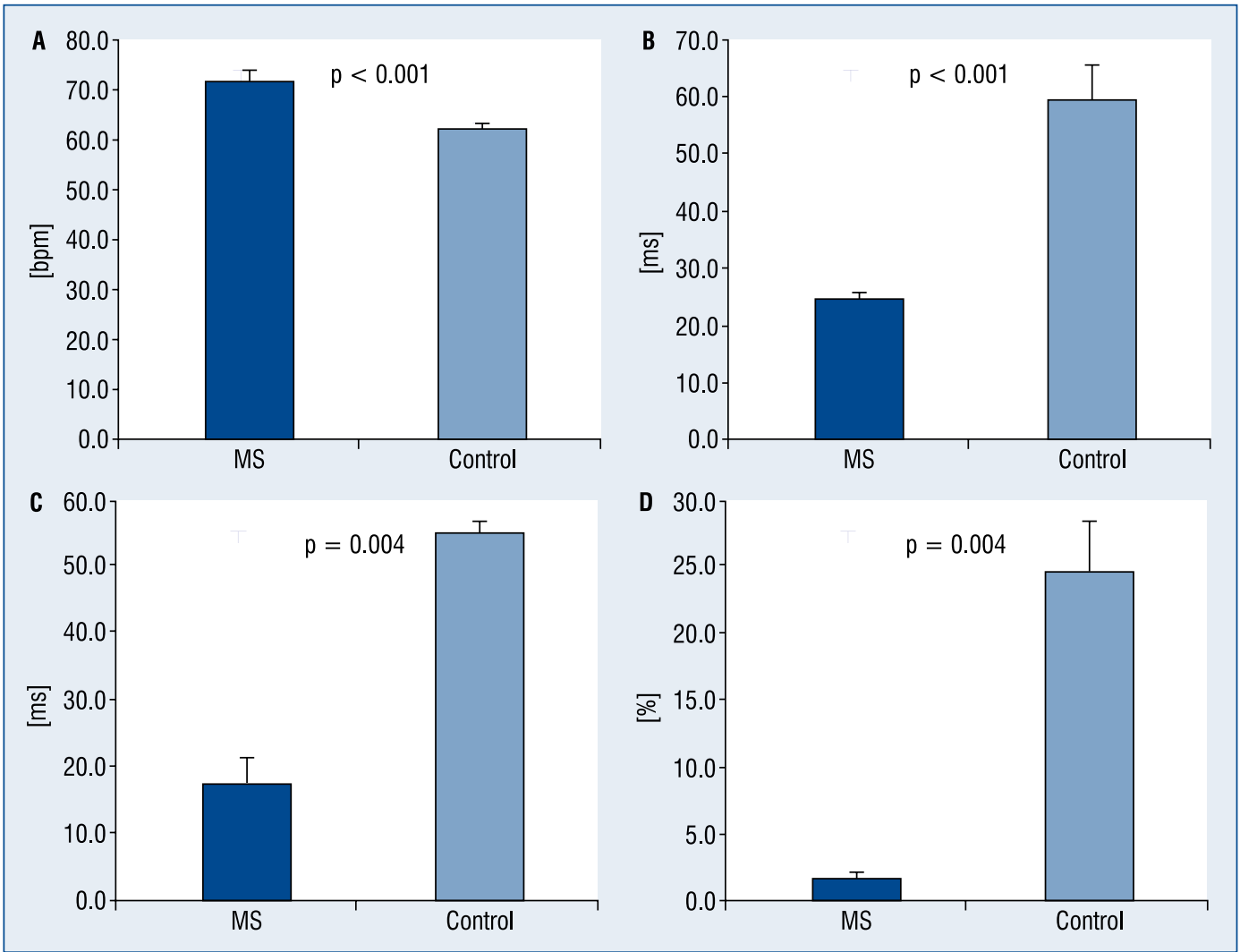

Figure 1. The values of heart rate (A), and time-domain indices of heart rate variability - standard deviation of normal RR intervals (SDNN) (B), square root of the mean squared differences of successive RR intervals (RMSSD) (C), and percentage of differences between adjacent normal RR intervals exceeding $50 \mathrm{~ms}$ (pNN50) (D) in 15 patients with FBN1 mutations and apparently healthy subjects (control group, $\mathrm{n}=30$ ) in supine position; MS - Marfan syndrome.

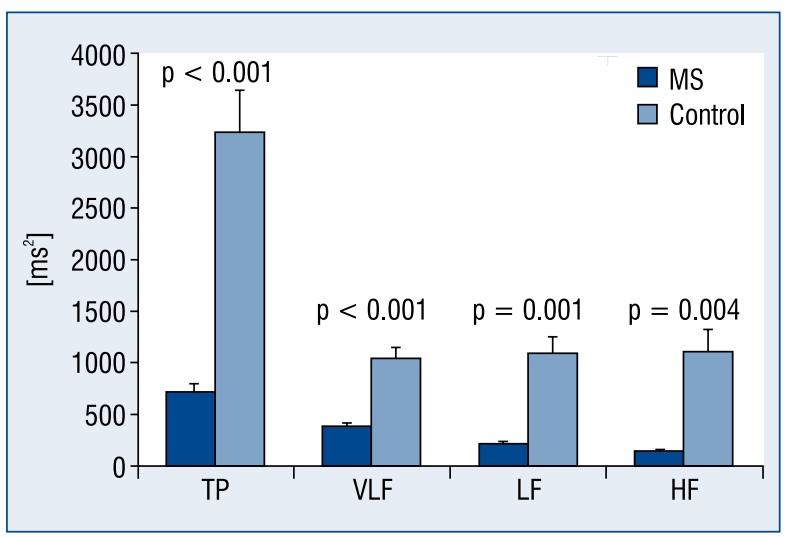

Figure 2. The values of frequency domain parameters of heart rate variability (total power [TP], very low frequency [VLF], low frequency [LF] and high frequency $[\mathrm{HF}])$ in 15 patients with FBN1 mutations and apparently healthy subjects (control group, $\mathrm{n}=30$ ) in supine position; MS - Marfan syndrome. lar to the findings in the supine position (Fig. 5). While in the OT of the control group, the value of VLF was about the same as LF. In MS patients, as was observed in the supine position, VLF waves dominated in the spectrum.

\section{HRV in MS patients with normal FBN1 gene}

Three patients with the MS phenotype were found to have a normal FBN1 gene. It was of interest to compare HRV in these patients with respective parameters in MS patients. The size of the sample was too small for providing sound statistical evaluation, however, these data still deserve to be described.

Figure 6 shows that all the patients with a normal FBN1 gene had higher SDNN values than any of the MS patients with the FBN1 mutation. SDNN is one of the most general and non-specific parameters of HRV and it correlates with many other parameters. Hence the mean value in this case seems to be much higher than in MS patients 


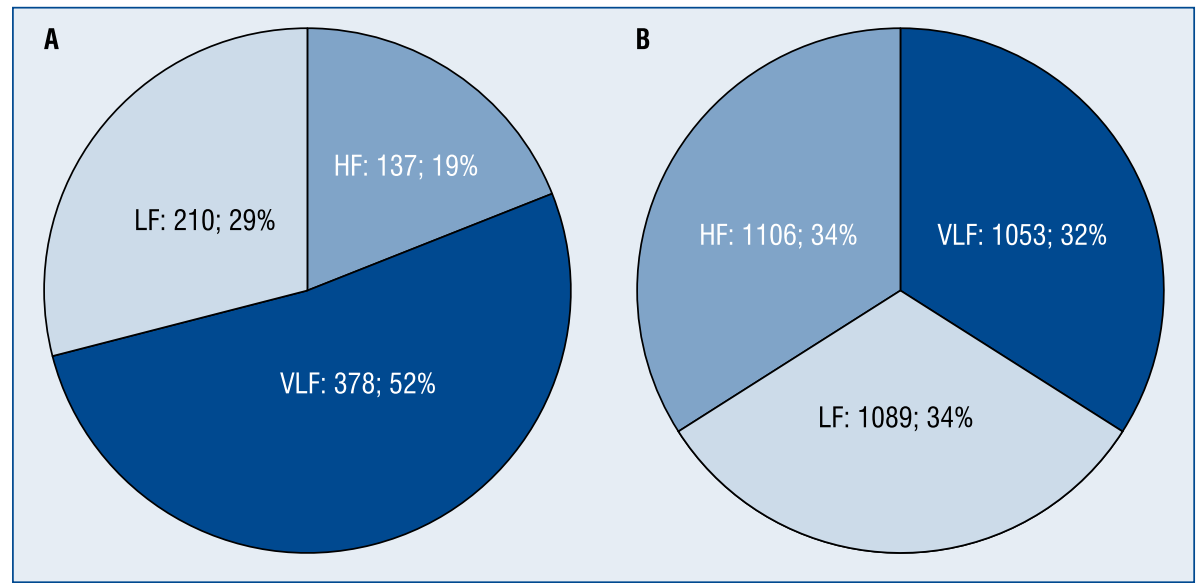

Figure 3. The absolute values and relative distribution of very low frequency (VLF), low frequency (LF), and high frequency (HF) power in 15 patients with FBN1 mutations $(A)$ and $(B)$ apparently healthy subjects (control group, $n=30$ ) in supine position. The values of the parameters (a power of respective band) is expressed in $\mathrm{ms}^{2}$.

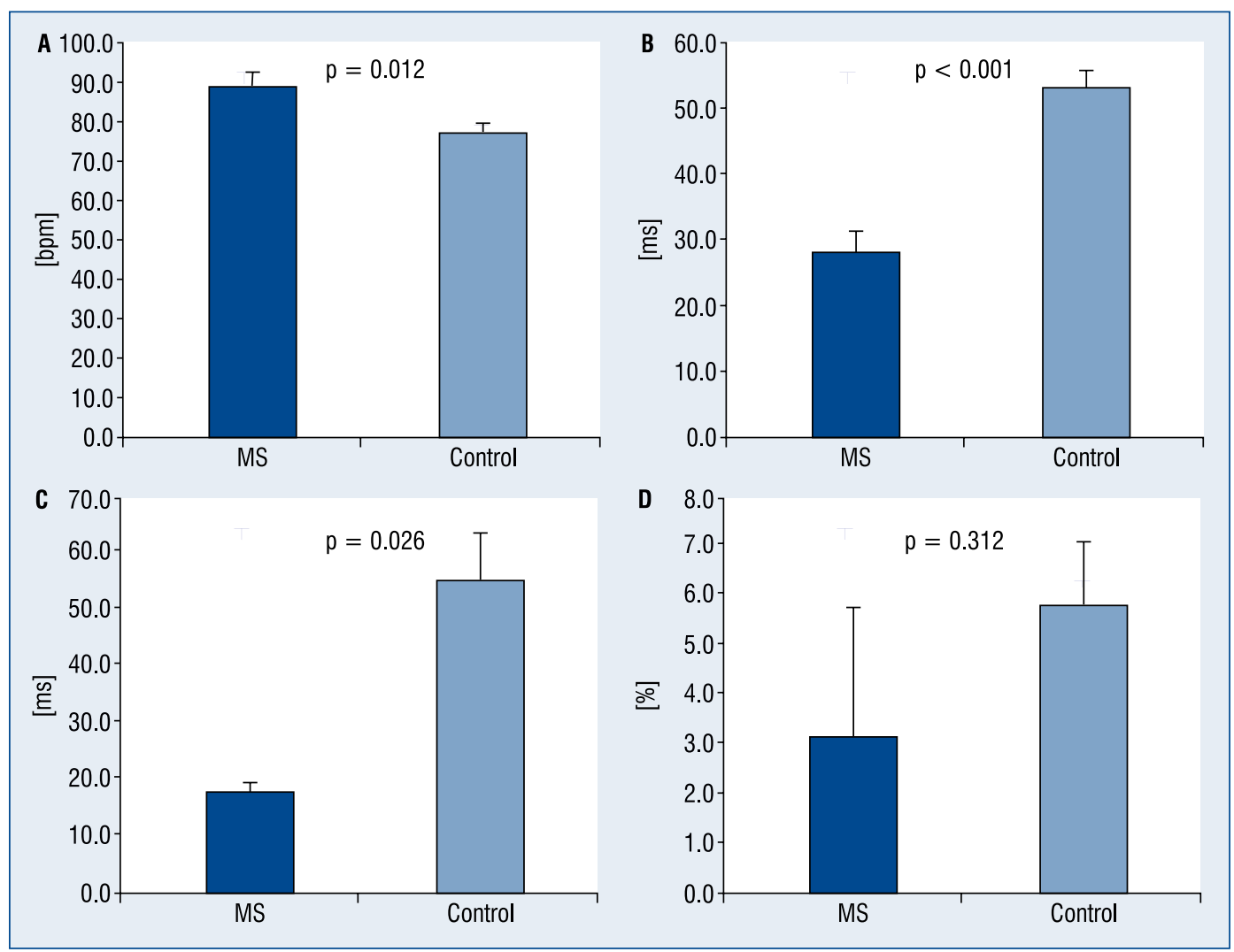

Figure 4. The values of heart rate (A), and time-domain indices of heart rate variability - standard deviation of normal RR intervals (SDNN) (B), square root of the mean squared differences of successive RR intervals (RMSSD) (C) and percentage of differences between adjacent normal RR intervals exceeding 50 ms (pNN50) (D) in 15 patients with FBN1 mutations and apparently healthy subjects (control group, $\mathrm{n}=30$ ) during orthostatic test; MS - Marfan syndrome.

with an FBN1 mutation (about $40 \mathrm{~ms}$ compared to $24 \mathrm{~ms}$ ), but it is still far below the value of healthy controls (59.4 ms).
In the case of spectral (frequency domain) HRV, a similar situation was observed (Supplementary Fig. S1). TP, LF and especially HF in 2 out of 


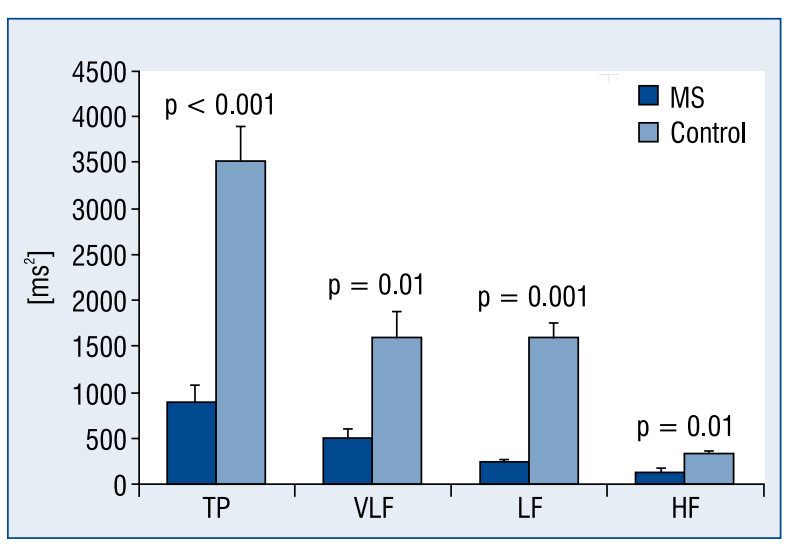

Figure 5. The values of frequency domain parameters of heart rate variability (total power [TP], very low frequency [VLF], low frequency [LF] and high frequency [HF]) in 15 patients with FBN1 mutations and apparently healthy subjects (control group, $\mathrm{n}=30$ ) during orthostatic test; MS - Marfan syndrome.

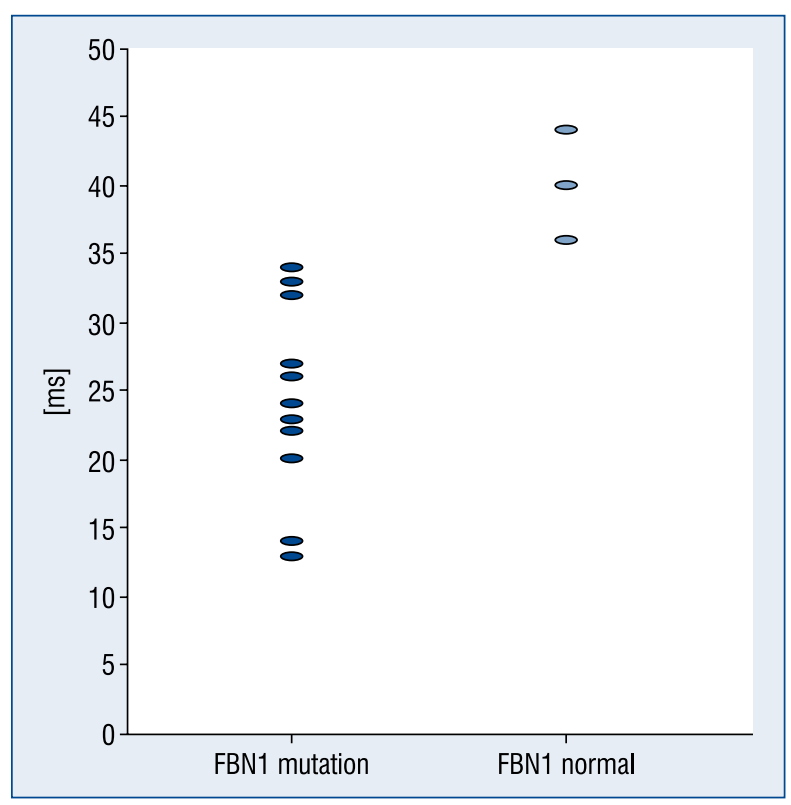

Figure 6. The values of standard deviation of normal $R R$ intervals in supine position in patients with $(n=15)$ and without FBN1 mutations $(n=3)$ in supine position.

3 patients without the FBN1 mutation were much higher than in MS patients with the FBN1 mutation, while one value was within the range of the least group. In case of VLF the trend was the same, but the difference was not so evident. These unexpected observations are precarious and may not be confirmed in further studies. However, this finding should not be ignored since it could be important for explaining further the possible mechanisms of decreased HRV in MS patients.

\section{Discussion}

Marfan syndrome has a special clinical pattern with typical "recognizable" anatomical features. However, development and availability of genetic testing revealed a number of syndromes which overlap with MS by clinical manifestations but differ by genetic defects. Since the molecular mechanism of these diseases is different, it could be expected that some of the clinical features and outcomes may be different too. This is particularly important in the case of serious complications and life-threatening conditions like aortic dissection and cardiac arrhythmias, because with similar clinical features the risk of mortality and serious complications could be completely different.

Obtained anthropometric data were usual the height was expectedly higher and consequently BMI lower in MS patients compared to the respective controls. Other parameters were similar in both groups. Clinical features of MS were comparable with the data from other authors [16]. However, a relatively large number of patients, almost half, underwent serious surgery - aortic root/valvereplacing surgery (Bentall procedure) in the past which may increase the severity of MS manifestations in our cohort. This may indicate that these patients have more severe manifestations of MS with the development of life threatening complications requiring surgical correction. Nevertheless, Bentall surgery, in particular its modified version, is considered reliable and relatively safe. Therefore, long-term outcomes are rather optimistic $[2,17]$. In our study, we did not observe substantial differences in HRV parameters of MS patients with FBN1 mutations after surgery compared to those who did not undergo the procedure. The distribution of HRV was similar in both subgroups and separate analysis has not been performed in this study.

The most important cardiovascular concerns after aortal dissections and aneurysms in patients with MS are ventricular arrhythmias [6]. It is well known that decreased HRV is associated with numerous health problems including increased allcause mortality, ventricular arrhythmia mortality, heart failure, diabetes mellitus type 2 risks even without evidence of autonomic neuropathy, liver damage, hypertension and many other problems [8]. There is growing evidence that a decrease in HRV eventually may appear not only as a conse- 
quence of decreased activities of sympathetic and parasympathetic branches of autonomic nervous system, but also as an independent metabolic phenomenon, related to possible impairment of cardiomyocytes, especially cells of sinoatrial node (SAN) that may be less responsive to the neural and humoral impacts [18].

The exact mechanism of HRV suppression in MS remains unclear. However, in our opinion, there exist at least several possibilities. First, a genetic mutation in FBN1 gene leads to changes responsible for HRV decrease. To our best knowledge, up to date there has been no experimental or clinical evidence of affected neurological function, especially function of autonomic nervous system that may decrease the oscillations of the heart rhythm. In MS patients, the TGF- $\beta$ pathway is overactivated and that may cause several important cardiovascular consequences. Studies on the rat model of pressure overload proved involvement of TGF- $\beta$ activation in myocardial fibrosis and diastolic dysfunction [5]. Importantly, this study has demonstrated that TGF- $\beta$ function blocking resulted in prevention of fibroblast activation (inhibition of fibrosis) and also prevented diastolic dysfunction development. TGF- $\beta$ might suppress HRV by activating inflammatory cascades, affecting endothelial function with inhibited nitric oxide release and development of oxidative stress [19].

In this study, among the patients with clinically diagnosed MS according to revised Ghent criteria, 15 subjects had confirmed FBN1 mutations. The other 3 patients who did not have FBN1 mutations were included into a separate small subgroup. Despite a low number of patients (only 3), dramatic increases in HRV were observed compared to patients with a confirmed mutation of FBN1 (Figs. 6, 7). It would probably be more correct to say partially preserved HRV rather than increased, considering higher values of HRV in the controls. These rather accidental findings indicate that FBN1 mutation and malfunction may be specifically responsible for HRV suppression in MS patients with FBN1 mutations, either directly or indirectly. However, the mechanisms that may cause the fibrillin 1 negative influence on HRV are unclear. First of all, fibrillin 1 is a protein of the extracellular matrix and it is difficult to imagine in what way its deranged function may influence activity of the autonomic nervous system. Besides, there is no evidence that functionally insufficient fibrillin 1 may affect the electric activities of neurons or cardiomyocytes. These observations, considering obvious differences between the groups, indicate rather indirect effects of this mutation.

An important pathogenic mechanism likely to be indirectly involved is an increased concentration of TGF- $\beta$ as a result of altered binding to the defective fibrillin 1 protein $[1,2]$. TGF- $\beta$, as shown in numerous studies, is responsible for vascular dysfunction and oxidative stress [19], myocardial fibrosis, diastolic dysfunction and other cardiovascular alterations $[2,4,5]$. The second possible mechanism of HRV suppression derives from the study of affected skeletal muscle function in MS patients. The decreased efficiency of an energy supply within the muscle cells was documented [20]. The study of biventricular dysfunction in MS by cardiac magnetic resonance revealed that it is an intrinsic feature not related to aortic elasticity or $\beta$-blocker usage [21]. This means that a specific problem of heart functioning, probably of metabolic nature exists in MS. This raises the other important suggestion that the HRV suppression is likely be due to the problems in intrinsic excitability and deranged properties of the SAN and its cells rather than suppressed autonomic regulation. Similarly, as in skeletal muscles, affected electrical activity and metabolic efficiency in the SAN cells may suppress their ability to respond to the external regulatory signals. We suggest that the amplitude of the heart rhythm oscillations will be higher when the adenosine thriphosphate (ATP) concentration in the cells is higher. More profound hyperpolarization of cell membrane and consequently lower amplitude is expected in conditions of metabolic problems such as low ATP supply due to acute or chronic hypoxia, derangements of redox potential and signaling [18].

The third possibility is that the decrease in HRV appears as a consequence of unspecific disease advancement. Chronic persistence of defective fibrillin 1 protein accompanied by the activation of TGF- $\beta$ pathway could lead to the chronic microand macrovascular dysfunction [19], gradual decrease in tissue perfusion efficiency, development of hypoxia and therefore increase in sympathetic activity, and consecutively elevation of HR. This possibility is also supported by the increased susceptibility of MS patients to the serious ventricular rhythm disorders including ventricular fibrillation. Low HRV is also known to be a risk factor for fatal ventricular arrhythmias, especially in case of myocardial infarction [8]. The medium for this seems to be local hypoxia and lack of ATP synthesis and respective energy supply for maintaining sufficient membrane potential of cardiac cells. 
Finally, the fourth possible mechanism of HRV suppression arises from the recent indications of oxidative stress development in MS patients [19]. A relatively simple mouse model, as well as cohort human study clearly showed the evidence of accumulation of carbonyl protein modification products in human serum and isoprostane accumulation and low superoxide dismutase expression in mouse model. The patients with the diseases typically considered to be associated with oxidative stress usually have severely impaired HRV. This does not seem to be a coincidence since this association is overwhelming, however the explanations and cause-consequence relationships remain largely unclear. In fact, there have been a few recent attempts to solve this puzzle, yetr the authors could not provide sufficient evidence and theoretical background for the explanation of this phenomenon [22]. As we have recently hypothesized [18], the interpretation of decreased HRV may not be simply limited to an autonomic dysfunction and increased sympathetic tone. The negative correlations of HRV parameters with weight, visceral fat, BMI, and a number of carbohydrate metabolism parameters in apparently healthy sedentary subjects also confirm this hypothesis [23]. The energetic deficiency as well as chronic hypoxia and persistence of oxidative stress seem to play major roles in HRV suppression in MS patients. In real life, all mentioned mechanisms plausibly contribute to HRV suppression in MS patients with FBN1 gene mutations. All these processes, to some extent, take place in the organism of patients and HRV decrease reflects the degree of metabolic derangements and heart tissue damage. The growing interest in HRV as the prognostic and risk stratification marker for cardiovascular diseases also appears to be important for MS [12]. Moreover, monitoring of HRV for a follow-up observation of disease progression could serve as a sensitive indicator reflecting dynamics of treatment efficiency [15].

\section{Limitations of the study}

The limitations of our study arise mainly from the relatively small cohort of patients recruited. Almost a half of the patients were post-surgery which means that they were at an advanced stage of the disease. However, the remaining patients also were not in the condition of good health. Some of the patients before participation in the study did not take losartan as a preventive medicine and were recommended to start the treatment as soon as possible. A larger number of patients with the MS phenotype but with a normal FBN1 gene could also improve the quality of the study. The measurement of plasma levels of TGF- $\beta$ and determination of possible correlations with HRV parameters may add important information for analysis and interpretation of mechanistic relationships. Nevertheless, statistical evaluation revealed convincing differences in the HRV between MS patients with FBN1 mutations and healthy controls, thus confirming these substantial changes point out potential clinical implications.

\section{Conclusions}

A marked decrease of HRV, documented in the study, may be an important clinical feature in MS patients with confirmed FBN1 gene mutations. Its potential role as a marker of disease severity and increased risk of mortality, independent of surgical issues, requires further careful evaluation.

\section{Highlights}

1. Marfan syndrome patients with FBN1 mutation have decreased HRV.

2. The reduction of HRV was observed for both time- and frequency-domain indices.

3. HRV decrease is likely to be caused by biochemical effects of defective fibrillin 1 protein.

4. Low HRV may contribute to the severity of the disease independent of vascular complications.

\section{Acknowledgements}

Authors are grateful to Prof. Bart Loeys and Dr Lut Van Laer from the Center of Medical Genetics, Antwerp, Belgium for genetic testing and Percy Herbert from Redlands, California, United States for valuable comments regarding the manuscript.

\section{Conflict of interest: None declared}

\section{References}

1. Jondeau G, Michel JB, Boileau C. The translational science of Marfan syndrome. Heart, 2011; 97: 1206-1214. doi: 10.1136/hrt.2010.212100.

2. Judge DP, Dietz HC. Marfan's syndrome. Lancet, 2005; 366 : 1965-1976. doi: 10.1016/S0140-6736(05)67789-6.

3. Loeys BL, Dietz HC, Braverman AC et al. The revised Ghent nosology for the Marfan syndrome. J Med Genet, 2010; 47: 476-485. doi: 10.1136/jmg.2009.072785.

4. Hao X, Zhang Y, Zhang X et al. TGF- $\beta 1$-mediated fibrosis and ion channel remodeling are key mechanisms in producing the sinus node dysfunction associated with SCN5A deficiency and aging. Circ Arrhythm Electrophysiol, 2011; 4: 397-406. doi: 10.1161/ /CIRCEP.110.960807.

5. Kuwahara F, Kai H, Tokuda K et al. Transforming growth factorbeta function blocking prevents myocardial fibrosis and diastolic 
dysfunction in pressure-overloaded rats. Circulation, 2002; 106: 130-135.

6. Savolainen A, Kupari M, Toivonen L, Kaitila I, Viitasalo M. Abnormal ambulatory electrocardiographic findings in patients with the Marfan syndrome. J Intern Med, 1997; 241: 221-226.

7. Task Force of the European Society of Cardiology and the North American Society of Pacing and Electrophysiology. Heart rate variability. Standards of measurement, physiological interpretation, and clinical use. Eur Heart J, 1996; 17: 354-381.

8. Xhyheri B, Manfrini O, Mazzolini M, Pizzi C, Bugiardini R. Heart rate variability today. Prog Cardiovasc Dis, 55: 321-331. doi: 10.1016/j.pcad.2012.09.001.

9. Papaioannou VE, Dragoumanis C, Theodorou V, Gargaretas C, Pneumatikos I. Relation of heart rate variability to serum levels of C-reactive protein, interleukin 6, and 10 in patients with sepsis and septic shock. J Crit Care, 2009; 24: 625.e1-e7. doi: 10.1016/j. jcrc.2008.11.010.

10. Pal GK, Adithan C, Ananthanarayanan PH et al. Sympathovagal imbalance contributes to prehypertension status and cardiovascular risks attributed by insulin resistance, inflammation, dyslipidemia and oxidative stress in first degree relatives of type 2 diabetics. PLoS One, 2013; 8: e78072. doi: 10.1371/journal. pone.0078072.

11. Aydin A, Adsay BA, Sheikhzadeh S et al. Observational cohort study of ventricular arrhythmia in adults with Marfan syndrome caused by FBN1 mutations. PLoS One, 2013; 8: e81281. doi: 10.1371/journal.pone.0081281.

12. Hoffmann BA, Rybczynski M, Rostock T et al. Prospective risk stratification of sudden cardiac death in Marfan's syndrome. Int J Cardiol, 2013; 167: 2539-2545. doi: 10.1016/j.ijcard.2012.06.036.

13. Van Dijk N, Immink RV, Mulder BJM, van Lieshout JJ, Wieling W. Orthostatic blood pressure control in Marfan's syndrome. Europace, 2005; 7: 25-27. doi: 10.1016/j.eupc.2004.05.009.

14. Van Dijk N, Boer MC, Mulder BJM, van Montfrans GA, Wieling W. Is fatigue in Marfan syndrome related to orthostatic intolerance? Clin Auton Res, 2008; 18: 187-193. doi: 10.1007/s10286--0080475-y.
15. Abrahamovych O, Cherkas A, Abrahamovych U, Abrahamovych M Serhiyenko V. Heart rate variability: Physiological bases, clinical importance and peculiarities in patients with peptic ulcer and after resection of stomach. In: Abrahamovych O ed. Danylo Halytskyi Lviv National Medical University, Lviv: 2014: doi: 10.13140/2.1.4866.8960.

16. Faivre L, Collod-Beroud G, Loeys BL et al. Effect of mutation type and location on clinical outcome in 1,013 probands with Marfan syndrome or related phenotypes and FBN1 mutations: An international study. Am J Hum Genet, 2007; 81: 454-466. doi: 10.1086/520125.

17. Tamura K, Arai H, Kawaguchi S et al. Long-term results of modified Bentall procedure using flanged composite aortic prosthesis. Ann Thorac Cardiovasc Surg, 2013; 19: 126-130.

18. Cherkas A, Yatskevych $O$. The amplitude of heart rate oscillations is dependent on metabolic status of sinoatrial node cells. OA Med Hypothesis, 2014; 2: 1-8.

19. Yang HHC, van Breemen C, Chung AWY. Vasomotor dysfunction in the thoracic aorta of Marfan syndrome is associated with accumulation of oxidative stress. Vascul Pharmacol, 52: 37-45. doi: 10.1016/j.vph.2009.10.005.

20. Crilley JG, Bendahan D, Boehm EA et al. Investigation of muscle bioenergetics in the Marfan syndrome indicates reduced metabolic efficiency. J Cardiovasc Magn Reson, 2007; 9: 709-717. doi: 10.1080/10976640701317028.

21. De Witte P, Aalberts JJJ, Radonic T et al. Intrinsic biventricular dysfunction in Marfan syndrome. Heart, 2011; 97: 2063—2068. doi: 10.1136/heartjnl-2011-300169.

22. Yelisyeyeva O, Cherkas A, Semen K, Kaminskyy D, Lutsyk A. Study of aerobic metabolism parameters and heart rate variability and their correlations in elite athletes: A modulatory effect of amaranth oil. Clin Exp Med J, 2009; 3: 293-307. doi: 10.1556/ /CEMED.3.2009.2.9.

23. Cherkas A, Abrahamovych O, Golota S et al. The correlations of glycated hemoglobin and carbohydrate metabolism parameters with heart rate variability in apparently healthy sedentary young male subjects. Redox Biol, 2015; 5: 301-307. doi: 10.1016/j. redox.2015.05.007. 


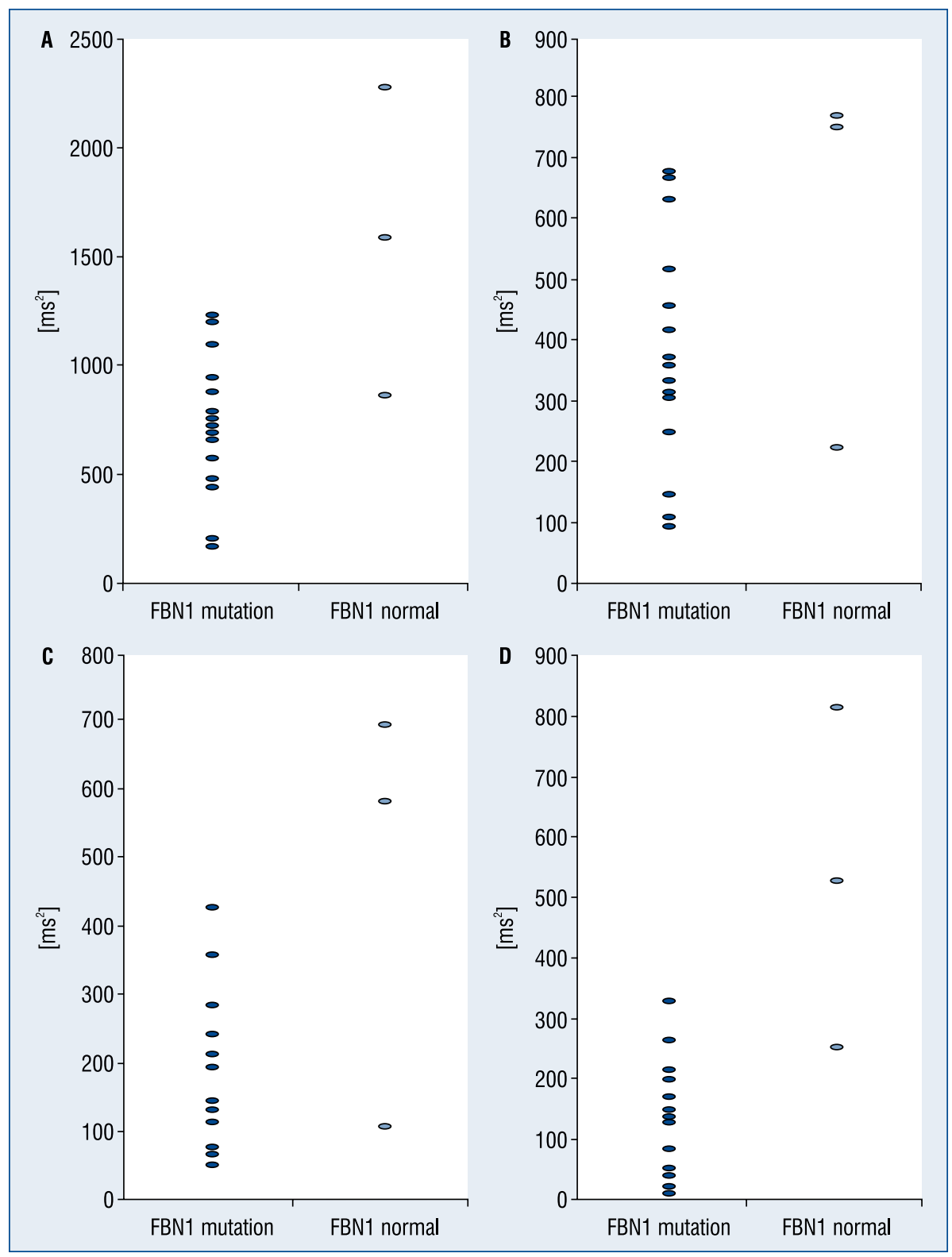

Supplementary Figure S1. The values of frequency domain parameters of heart rate variability: total power (TP) (A), very low frequency (VLF) (B), low frequency (LF) (C) and high frequency (HF) (D) in patients with $(n=15)$ and without FBN1 mutations $(n=3)$ in supine position. 